\title{
ORIGINAL
}

\section{FIABILIDAD Y VALIDEZ DE UN CUESTIONARIO PARA MEDIR EN ESTUDIANTES UNIVERSITARIOS LA ASOCIACIÓN DE LA ANSIEDAD Y DEPRESIÓN CON FACTORES ACADÉMICOS Y PSICOSOCIOFAMILIARES DURANTE EL CURSO 2004-2005}

\author{
Serafín Balanza Galindo, Isabel Morales Moreno, Joaquín Guerrero Muñoz, Ana Conesa Conesa \\ Universidad Católica San Antonio de Murcia.
}

RESUMEN

Fundamento: La elevada frecuencia de ansiedad y depresión en estudiantes universitarios está relacionada con factores sociales, familiares y con el estrés académico. El objetivo de este estudio ha sido determinar la consistencia interna y la validez de un cuestionario de situaciones socio familiares y académicas que pueden estar relacionadas con situaciones psicopatológicas en estudiantes universitarios

Método: El estudio se realizó en la Universidad Católica San Antonio de Murcia, en una muestra de 700 alumnos a los que se les administró un cuestionario de elaboración propia, que contenía ítems que valoraban aspectos académicos y socio familiares. Como factores de estudio independientes se utilizaron variables que medían diversas situaciones estresantes de los estudiantes y la escala de ansiedad y depresión de Goldberg, todo con el fin de medir la validez del cuestionario.

Resultados: La fiabilidad del cuestionario quedó demostrada al obtener en el test-retest un coeficiente de correlación intraclase de 0,819. El cuestionario original de 19 ítems quedó reducido a 15 tras el análisis de homogeneidad, obteniendo un alfa de Cronbach de 0,758. La validez de constructo se evaluó con el análisis factorial del cuestionario, resultando dos factores que representaban a "aspectos académicos" y "aspectos sociofamiliares". Los estudiantes con el test de ansiedad y depresión positivo eran los que más puntuación obtuvieron en el cuestionario global y en los dos factores, quedando demostrada la validez de criterio

Conclusiones: El cuestionario del estudio es una herramienta de valoración, de fácil uso y de ágil aplicación para la detección precoz de ansiedad y depresión en estudiantes universitarios

Palabras clave: Estudiantes. Ansiedad. Depresión. Cuestionario. Reproducibilidad de Resultados.

Correspondencia:

Serafín Balanza Galindo

Universidad Católica San Antonio de Murcia

Guadalupe. 30107 Murcia

Correo electrónico: balanza2@yahoo.es
ABSTRACT

\section{Academic and Psycho-socio-familiar Factors Associated to Anxiety and Depression in University Students. Reliability and Validity of a Questionnaire}

Background: The high frequency of anxiety and depression in university students is related to social, family factors and academic stress. The aim of this research is to determine the internal consistency and validity of a questionnaire on sociofamiliar and academic situations which may be related to psychopathological situations in university students.

Methods: The research was carried out at the Universidad Católica San Antonio de Murcia with 700 students, to whom a questionnaire made by the researchers was given. This questionnaire included items which evaluated academic and socio-familiar aspects. Variables regarding various stressful situations amongst students, and the Goldberg's level of anxiety and depression scale were used as independent facts of research in order to measure the validity of the questionnaire.

Results: The reliability of the questionnaire was shown after obtaining an intraclass correlation coefficient of 0.819 . The original questionnaire with 19 items was reduced to 15 items after the homogeneity analysis, obtaining a Cronbach alpha of 0.758 . The validity of constructio was evaluated with the factor analysis of the questionnaire, with a result of two factors which represented 'academic aspects' and 'socio-familiar aspects'. Those students with a positive anxiety and depression test were the ones who obtained the higher score on the global questionnaire and in both factors, proving the validity of the criteria.

Conclusions: The research questionnaire is an agile and easy to use tool for the assessment and early detection of anxiety and depression in university students.

Key words: Students. Anxiety. Depression. Questionnaire. Reproducibility of Results. 


\section{INTRODUCCIÓN}

Los estudios que han relacionado la ansiedad y la depresión con factores psicosociales y familiares a los que el individuo se ve expuesto a lo largo de la vida son abundantes en la literatura científica, así como aquellos que se refieren al estrés académico y su repercusión en el estado de salud de los estudiantes universitarios $^{1-4}$.

La ansiedad y la depresión son los desórdenes clínicos más frecuentes en la población en general que también se encuentran significativamente presentes entre los estudiantes universitarios ${ }^{5,6}$. El inicio, el desarrollo y la duración de estas psicopatologías pueden estar relacionados con la presencia de múltiples factores, tanto situacionales como psicológicos.

La literatura científica en este campo viene describiendo la importancia que determinados factores personales y psicosociales poseen en la aparición y el desarrollo de trastornos psicológicos entre la población de estudiantes universitarios. El contexto familiar se ha revelado como un indicador sobresaliente del bienestar emocional y del ajuste psicológico del estudiante. Las relaciones con los padres, los vínculos familiares, los estilos educati$\operatorname{vos}^{7,8}$, la crisis de pareja y la discrepancia conyugal-parental expresada en situaciones de ruptura como la separación o el divorcio ${ }^{9,10}$, la comunicación y la cohesión-conflictividad familiar e intergeneracional $^{11,12}$ o la psicopatología parental previa $^{13-16}$ son variables que se han vinculado al estado de salud mental de los estudiantes universitarios y de los jóvenes en general. Por descontado, los factores sociales y ambientales han sido también objeto de análisis, especialmente aquéllos que modulan el nivel de estrés al que los estudiantes universitarios se ven expuestos a lo largo de su carrera. El desarrollo de habilidades y competencias para resol- ver los problemas cotidianos ${ }^{17}$, las relaciones positivas con compañeros y profesores y el afrontamiento eficaz de las transiciones de la vida y los life events ${ }^{18-21}$ se han destacado como variables que disminuyen el estrés y el riesgo de sufrir un trastorno psicológico. Otros estudios han abordado el papel que la carga de trabajo y los recursos económicos tienen sobre la salud mental y física de los estudiantes indicándonos que si bien poseen un impacto evidente no son tan decisivos como otros factores que ya se han indicado y que, en cualquier caso, su relación con otras prácticas y hábitos como el consumo de alcohol o drogas explicaría en mayor grado su influencia en el detrimento de la salud de los estudiantes ${ }^{22,23}$. Los trabajos de investigación en este terreno indican la importancia que tienen sobre el estado de salud mental y viceversa considerar la influencia de determinados hábitos y conductas. Los trastornos de ansiedad y depresión poseen una comorbilidad psiquiátrica elevada con el consumo de alcohol y drogas. En muchos casos la ansiedad y la depresión aumentan la sensibilidad y la predisposición al consumo de sustancias adictivas altamente perjudiciales para la salud que acaban deteriorando las relaciones sociales ${ }^{24-28}$.

Este trabajo ha consistido en la elaboración de un cuestionario ad hoc que nos permitiera identificar y discriminar algunos de los más representativos, y que claramente pudiéramos vincular al estado de salud mental de los estudiantes universitarios. Para ello formulamos una serie de preguntas o ítems y empleamos la Escala de Ansiedad y Depresión de Goldberg (EADG) con el propósito de valorar la existencia de indicadores clínicos relevantes. En concreto, usando como criterio el valor predictivo de los índices de la EADG, se ha puesto a prueba una serie de cuestiones en las que se consideran aspectos académicos, como la satisfacción con el sistema de enseñanza, el ren- 
dimiento, las expectativas de éxito, la motivación y la ansiedad ante los exámenes, y aspectos del entorno social y familiar como la convivencia en el lugar de residencia durante los estudios universitarios, la relación de pareja o el apoyo percibido.

El objetivo de este estudio ha sido determinar la consistencia interna y la validez de un cuestionario de situaciones socio familiares y académicas que pueden estar relacionadas con situaciones psicopatológicas en estudiantes universitarios.

\section{SUJETOS Y MÉTODOS}

Con los objetivos propuestos se ha diseñado un estudio con metodología cuantitativa, observacional, descriptivo y transversal

Población. La población diana objeto de este estudio esta formada por los 4.821 estudiantes matriculados en la Universidad Católica San Antonio de Murcia, durante el curso 2004-2005, en titulaciones oficiales, excluyendo a los alumnos de cursos de postgrado y master.

Tamaño de la muestra y muestreo. El cálculo de la muestra se ha realizando en base a estimación, por estudios previos ${ }^{29,30}$, de que el $35 \%$ de los estudiantes universitarios sufren algún trastorno psicopatológico, con una precisión de $\pm 3,5 \%$ y un error $\alpha$ de $5 \%$ y para poblaciones finitas, resultando una muestra necesaria de 622 alumnos, pero teniendo en cuenta una perdida del $10 \%$, por las encuestas no válidas, se estimó una muestra de 684 alumnos.

Se ha utilizado una técnica de muestreo aleatorio multietápico por conglomerados, siendo la última unidad de muestreo el curso, realizando el cuestionario todos los alumnos que asistían a clase en el momento de la entrevista. El trabajo de campo se realizó durante el tercer trimestre de curso académico 2004-2005. La muestra ha sido distribuida por Facultades o Escuelas y para que fuese representativa ha sido estratificada en función de los alumnos matriculados.

Variables e instrumento de medida. Para la realización del estudio se ha utilizado un cuestionario de elaboración propia y como factores de estudio independientes las variables: sexo, Facultad o Escuela Universitaria, ¿realiza largos desplazamientos para acudir a la universidad ( $>50 \mathrm{Kms})$ ?, compaginar estudios y trabajo, tener otras obligaciones además de estudiar (cuidar a hijos, familiares, etc.), tener asignaturas pendientes del curso pasado y residir con su familia durante el curso académico.

El cuestionario de elaboración propia fue creado para medir los aspectos negativos de un estudiante universitario que pudieran estar asociados con cuadros clínicos de ansiedad o depresión, con 19 ítems, todas las preguntas fueron formuladas en sentido negativo, medidas en escala de Likert, con 5 posibles respuestas, donde el valor 1 es total desacuerdo y el valor 5 al total acuerdo. Los ítems del cuestionario desarrollado para este estudio se redactaron en base a la revisión bibliográfica pero, sobre todo, están basados en las observaciones dadas por los alumnos en las reuniones mantenidas con ellos y por la experiencia docente del equipo investigador, construyéndose un cuestionario que agrupase factores negativos relacionados con aspectos académicos, sociales, familiares y personales.

Procedimiento y análisis de resultados. Se han seguido los pasos metodológicos necesarios para analizar la fiabilidad y validez de un cuestionario.

Prueba piloto: Para comprobar la compresión de los distintos ítems que componía la escala y verificar la fiabilidad de la 
misma, se realizó una prueba piloto, siguiendo el criterio de que el número de sujetos participantes en esta prueba ha de ser siempre superior al número de ítems del cuestionario, recomendando una participación de sujetos entre dos y diez veces el número de ítems ${ }^{31}$. En la muestra piloto del estudio participaron 38 estudiantes, sin ningún criterio de muestreo, simplemente que cumpliesen los criterios necesarios para poder participar en el estudio. Para evaluar la repetibilidad del cuestionario se realizo el test-retest al grupo que participaron en la prueba piloto, repitiendo el cuestionario a los 15 días de haber realizado el primero, calculándose el coeficiente de correlación intraclase (CCI), con el objetivo de medir el nivel concordancia entre las medidas cuantitativas del cuestionario, considerando como aceptable una CCI superior a $0,70^{32}$.

Fiabilidad y homogeneidad del cuestionario: La consistencia interna u homogeneidad entre los ítems del cuestionario se realizó aplicando el coeficiente alfa de Cronbach $^{33}$, cuyos valores oscilan entre $0 \mathrm{y}$ 1, considerando como aceptable valores iguales o superiores a $0,70^{33,34}$, evaluando si su valor aumentaba o disminuía al extraer del cuestionario cada uno de los ítems que lo componen. También se calcularon los coeficientes de correlación de cada ítem con los valores del cuestionario total-corregido, es decir, una vez extraído del cuestionario o de la escala el valor del ítem que se pretende correlacionar, eliminando del cuestionario aquellos de baja correlación con el total-corregido, cuando esta era inferior a $0,30^{35}$, colaborando de forma automática a un aumento del valor del alfa de Cronbach.

Validez de constructo: Para verificar la validez de constructo y analizar las dimensiones subyacentes existentes en el cuestionario se utilizó la prueba estadística multivariante de Análisis Factorial, verificando previamente si su utilización era adecuada mediante el Índice de Kaiser-Meyer-Olkin y el contraste de la matriz de correlaciones mediante el test de esfericidad de Barlett. El análisis factorial se realizó por el procedimiento de exploración de componentes principales de la matriz de correlación de todos los ítems del cuestionario, con rotación ortogonal utilizando el procedimiento Varimax y el criterio de Kaiser, extrayendo solamente los factores con un autovalor mayor de 1 , que son los que explican el mayor porcentaje de la variabilidad total. Para que los pesos factoriales fuesen consistentes, se ha establecido como criterio para que un ítem forme parte del factor extraído tener un valor superior o igual a $0,40^{36}$.

Validez de criterio: Para determinar la validez de criterio, en la forma de validez convergente, se ha tomado como referencia el cuestionario de Goldberg ${ }^{37,38}$ con una escala de ansiedad y otra para la depresión, totalmente independientes, administrados ambos cuestionarios de formas simultánea; en cada una de las subescalas existen 4 ítems iniciales de despistaje para determinar si es o no probable que exista un trastorno mental, y un segundo grupo de 5 ítems que se formulan sólo si se obtienen respuestas positivas a las preguntas de despistaje (2 o más en la subescala de ansiedad, 1 o más en la subescala de depresión). Los puntos de corte utilizados en este estudio han sido de $\geq 5$ para la escala de ansiedad, $\mathrm{y} \geq 3$ para la de depresión. Se analizó la correlación existente entre la puntuación del cuestionario y los valores del cuestionario de Goldberg, subescala de ansiedad y depresión, utilizando el coeficiente de correlación de Pearson. En la evaluación de la validez de hipótesis, con las variables independientes, se utilizaron la T de Student para muestras independientes y el análisis de la varianza para un factor.

El análisis se realizó con el paquete SPSS y la significación estadística se acepto para $\mathrm{p}<0,05$. 


\section{RESULTADOS}

El cuestionario fue cumplimentado por 700 alumnos con una edad media de 21,4 $\pm 2,9$ (IC 95\% 21,2-21.7) años, con un rango de valores que varían desde los 18 años hasta los 36 años. El 48,9\% fueron hombres y el $51,1 \%$ restante mujeres. En función a la Facultad o escuela Universitaria $176(25,1 \%)$ son alumnos de Ciencias Sociales y Comunicación, 109 (15,6\%) pertenecen a Ciencias Jurídicas y de la Empresa, $190(27,1 \%)$ a la Escuela Politécnica y $225(32,1 \%)$ a Ciencias de la Salud y del Deporte.

Con relación a las variables utilizadas como factores de estudio 324 (46,3\%) alumnos contestan tener que hacer un desplazamiento superior a 50 kilómetros para acudir diariamente a la Universidad, $207(29,6 \%)$ compaginan estudios y trabajo, $90(12,9 \%)$ contestan afirmativamente que tienen otras obligaciones, como cuidar a hijos o familiares, 230 (33\%) tienen alguna asignatura pendiente del curso anterior y $258(39,8 \%)$ residen durante el curso académico fuera del hogar familiar.

La Escala de Ansiedad y Depresión de Goldberg (EADG), utilizada en este estudio para el análisis de convergencia del cuestionario de elaboración propia, ha detectado, de forma global, y siempre haciendo mención a "probables casos", ya que es un instrumento de cribaje pero no de diagnóstico, que el $65,9 \%$ de los estudiantes universitarios entrevistados sufren probablemente un trastorno de ansiedad y / o de depresión. Con un punto de corte de $=5$ puntos para la escala de ansiedad, han resultado positivos $330(47,1 \%)$ de loa alumnos. En la escala de de depresión, con una puntuación de corte de $=3$ puntos, han resultado positivos $375(55,6 \%)$ de los alumnos encuestados.

\section{Cuestionario de elaboración propia}

Prueba piloto: En el análisis global del cuestionario, formado originariamente por 19 ítems, resultó una $\alpha$ alfa de Crombach de 0,845 . Después de suprimir dos ítems, por presentar una correlación con el total de la escala corregida muy débil, $<0,300$, la $\alpha$ de Crombach adquirió el valor de 0,871, mejorando considerablemente la consistencia interna y homogeneidad de los ítems del cuestionario.

La fiabilidad del cuestionario con el método del test-retest fue evaluada a partir de las puntuaciones totales obtenidas de la misma persona en dos ocasiones, con dos semanas de diferencia, participando 20 sujetos de los 36 iniciales, resultando bastante significativa al obtener un coeficiente de correlación intraclase, el total del cuestionario, de 0,819 (IC $95 \% 0,589-0,929), \mathrm{p}<0,001$.

Fueron eliminados del cuestionario original otros dos ítems; su eliminación no suponía una mejora de la consistencia interna, no mejoraba la $\alpha$ de Crombach, sino basado en criterios de validez lógica ambos ítems guardaban poca relación con el resto de la preguntas del cuestionario, considerando que no medían lo que realmente teníamos como objetivo medir.

Análisis de homogeneidad y selección de los ítems de la escala definitiva: El cuestionario, que tras la prueba piloto quedó reducido a 15 ítems, obtuvo un alfa de Crombach de 0,758 , obteniendo una media de $34,5 \pm 8,4$ puntos, siendo eliminados en un primer paso los ítems que no cumplían los requisitos de tener una correlación $>0,300$ con el valor total de la escala, los ítems $\mathrm{n}^{\circ} 13,15$ y 19 (tabla 1). Tras esta eliminación, se volvió a analizar la escala con los 12 ítems restantes, resultando un alfa de Crombach de 0,766, apareciendo el ítem $\mathrm{n}^{\circ} 17$ con una correlación con el total de la escala de 0,287 , siendo eliminado del cuestionario, quedando el cuestionario definitivo formado por 11 ítems, con un valor alfa de Crombach de 0,762. 
Tabla 1

Análisis de homogeneidad o consistencia interna de la escala original tras la realización de la prueba piloto

\begin{tabular}{|l|c|c|}
\hline \multicolumn{1}{|c|}{ ITEMS } & $\begin{array}{c}\text { Media } \\
\pm \\
\text { Desviación Típica }\end{array}$ & $\begin{array}{c}\text { Correlación ítem } \\
\text { con total escala }\end{array}$ \\
\hline 9. Me considero una carga económica para mi familia & $2,91 \pm 1,37$ & 0,318 \\
\hline 10. Me siento incomprendido por mi familia & $1,7 \pm 1,09$ & 0,418 \\
\hline 11. Tengo problemas interpersonales con amigos, compañeros & $1,54 \pm 0,91$ & 0,392 \\
\hline 12. Mi estado físico o de salud no es muy favorable actualmente & $2,03 \pm 1,22$ & 0,372 \\
\hline 13.Me preocupa la perdida y deterioro de valores en la sociedad & $3,30 \pm 1,24$ & 0,108 \\
\hline 14.No soy capaz de dar la talla, no cubro expectativas & $1,94 \pm 1,06$ & 0,498 \\
\hline 15. Soy introvertido y no tengo facilidad para las relaciones & $1,87 \pm 1,07$ & 0,266 \\
\hline 16. Tengo problemas personales con mi pareja & $1,73 \pm 1,14$ & 0,314 \\
\hline 17. Mi futuro profesional al terminar los estudios es preocupante & $2,37 \pm 1,29$ & 0,306 \\
\hline 18. La carrera que estoy realizando no me satisface plenamente & $1,75 \pm 1,13$ & 0,355 \\
\hline 19. Me falta tiempo para estudiar & $2,96 \pm 1,36$ & 0,234 \\
\hline 20. Estoy descontento con mi rendimiento académico & $2,67 \pm 1,21$ & 0,500 \\
\hline 21. Estoy poco satisfecho con la enseñanza recibida & $2,50 \pm 1,11$ & 0,381 \\
\hline 22. Rindo mal en los exámenes porque me pongo nervioso & $2,56 \pm 1,25$ & 0,462 \\
\hline 23. Me falta motivación para estudiar & $2,67 \pm 1,33$ & 0,448 \\
\hline \multicolumn{2}{|}{ Alfa de Crombach: 0,758 } & \\
\hline
\end{tabular}

Validez de constructo del cuestionario definitivo, análisis factorial: Para analizar la validez de constructo, inicialmente se recurrió al análisis factorial de la escala definitiva, verificando antes que sí reunía los criterios necesarios para poder realizarlo, mediante el test de Kaiser-Meyer-Olkin, que obtuvo un valor de 0,834 , y el test de esfericidad de Bartlett, que resultó estadísticamente significativo, $\mathrm{p}<0,001$. Este análisis demostró una estructura subyacente compuesta por dos factores, que siguiendo los criterios de Kaiser superaban valores propios mayores de 1 , los cuales, en conjunto, explicaban el 40,9\% de la varianza total de los resultados, el factor 1 la variabilidad del $30,00 \%$ y el factor 2 el $10,94 \%$, siendo la carga factorial de cada ítem en los factores totalmente satisfactoria para incorporarlo al modelo por presentar un valor $>0,40$. Tras la rotación, el factor 1 incluyó 6 ítems relacionados con "aspectos académicos" y el factor 2 formado por 5 ítems relacionados con "aspectos sociales y personales”, (tabla 2) (Anexo 1).

Tabla 2

Análisis factorial de la escala final: matriz de componentes rotados

\begin{tabular}{|c|c|c|}
\hline ITEMS & Factor 1 & Factor 2 \\
\hline 9. Me considero una carga económica para mi familia & & 0,406 \\
\hline 10. Me siento incomprendido por mi familia & & 0,573 \\
\hline 11. Tengo problemas interpersonales con amigos, compañeros & & 0,689 \\
\hline 12. Mi estado físico o de salud no es muy favorable actualmente & & 0,617 \\
\hline 14. No soy capaz de dar la talla, no cubro expectativas & 0,532 & \\
\hline 16. Tengo problemas personales con mi pareja & & 0,662 \\
\hline 18. La carrera que estoy realizando no me satisface plenamente & 0,539 & \\
\hline 20. Estoy descontento con mi rendimiento académico & 0,730 & \\
\hline 21. Estoy poco satisfecho con la enseñanza recibida & 0,656 & \\
\hline 22. Rindo mal en los exámenes porque me pongo nervioso & 0,456 & \\
\hline 23. Me falta motivación para estudiar & 0,705 & \\
\hline $\begin{array}{r}\text { Autovalores } \\
\text { Varianza }\end{array}$ & $\begin{array}{r}3,301 \\
30,0 \%\end{array}$ & $\begin{array}{r}1,203 \\
10,9 \%\end{array}$ \\
\hline
\end{tabular}


Anexo 1

Cuestionario definitivo tras la validación

\begin{tabular}{|c|c|c|c|c|c|}
\hline ITEMS & 1 & 2 & 3 & 4 & 5 \\
\hline \multicolumn{6}{|c|}{ Subescala "Aspectos académicos" } \\
\hline \multicolumn{6}{|l|}{ 14. No soy capaz de dar la talla, no cubro expectativas } \\
\hline \multicolumn{6}{|l|}{ 18. La carrera que estoy realizando no me satisface plenamente } \\
\hline \multicolumn{6}{|l|}{ 20. Estoy descontento con mi rendimiento académico } \\
\hline \multicolumn{6}{|l|}{ 21. Estoy poco satisfecho con la enseñanza recibida } \\
\hline \multicolumn{6}{|l|}{ 22. Rindo mal en los exámenes porque me pongo nervioso } \\
\hline \multicolumn{6}{|l|}{ 23. Me falta motivación para estudiar } \\
\hline \multicolumn{6}{|c|}{ Subescala "Aspectos socio familiares" } \\
\hline \multicolumn{6}{|l|}{ 9. Me considero una carga económica para mi familia } \\
\hline \multicolumn{6}{|l|}{ 10. Me siento incomprendido por mi familia } \\
\hline \multicolumn{6}{|l|}{ 11. Tengo problemas interpersonales con amigos, compañeros } \\
\hline \multicolumn{6}{|l|}{ 12. Mi estado físico o de salud no es muy favorable actualmente } \\
\hline 16. Tengo problemas personales con mi pareja & & & & & \\
\hline
\end{tabular}

Volviendo a analizar la homogeneidad, la subescala "aspectos académicos" obtiene un alfa de Crombach de 0,711 y la de "socio familiares" un alfa de Crombach de 0,608, teniendo todos los ítems un coeficiente de correlación con el total de la subescala superior a 0,300, excepto el ítem 9 con un coeficiente de 0,292 (tabla 3).

En el análisis de validez de constructo por hipótesis, en la tabla 4 aparecen las puntuaciones de las subescalas y escala total, en función de los factores de estudio independientes utilizados en el estudio. Hemos encontrado diferencias en la variable que clasifica a los alumnos según compaginen los estudios con el trabajo o no, de tal modo que los no trabajadores presentan puntuaciones medias mayores en la escala total y en las dos subescalas, resultando estadísticamente significativa en la escala total y la subescala de aspectos académicos. Del mismo modo, los alumnos que tienen otras obligaciones añadidas al estudio y los

Tabla 3

Análisis de homogeneidad o consistencia interna de la escala original tras la realización de la prueba piloto

\begin{tabular}{|c|c|c|}
\hline ITEMS & $\begin{array}{c}\text { Media } \\
\pm \\
\text { Desviación Típica }\end{array}$ & $\begin{array}{l}\text { Correlación ítem } \\
\text { con total escala }\end{array}$ \\
\hline \multicolumn{3}{|c|}{$\begin{array}{c}\text { Factor 1: Subescala "Aspectos académicos" Media:14,09 } \pm 4,5 \\
\text { Alfa de Crombach: } 0,711\end{array}$} \\
\hline 14. No soy capaz de dar la talla, no cubro expectativas & $1,94 \pm 1,06$ & 0,474 \\
\hline 18. La carrera que estoy realizando no me satisface plenamente & $1,75 \pm 1,13$ & 0,344 \\
\hline 20. Estoy descontento con mi rendimiento académico & $2,67 \pm 1,21$ & 0,557 \\
\hline 21. Estoy poco satisfecho con la enseñanza recibida & $2,50 \pm 1,11$ & 0,400 \\
\hline 22. Rindo mal en los exámenes porque me pongo nervioso & $2,56 \pm 1,25$ & 0,392 \\
\hline 23. Me falta motivación para estudiar & $2,67 \pm 1,33$ & 0,501 \\
\hline \multicolumn{3}{|c|}{$\begin{array}{c}\text { Factor 2: Subescala “Aspectos socio familiares" Media: } 9,99 \pm 3,5 \\
\text { Alfa de Crombach: } 0,608\end{array}$} \\
\hline 9. Me considero una carga económica para mi familia & $2,91 \pm 1,37$ & 0,292 \\
\hline 10. Me siento incomprendido por mi familia & $1,78 \pm 1,09$ & 0,404 \\
\hline 11. Tengo problemas interpersonales con amigos, compañeros & $1,54 \pm 0,91$ & 0,394 \\
\hline 12. Mi estado físico o de salud no es muy favorable actualmente & $2,03 \pm 1,22$ & 0,350 \\
\hline 16. Tengo problemas personales con mi pareja & $1,73 \pm 1,14$ & 0,349 \\
\hline Media: $24,08 \pm 7,0$ & ach: 0,762 & \\
\hline
\end{tabular}


Tabla 4

Valores medios de la escala y subescala en función de las distintas variables introducidas en el estudio

\begin{tabular}{|c|c|c|c|}
\hline & Aspectos académicos & $\begin{array}{l}\text { Aspectos socio } \\
\text { familiares }\end{array}$ & Escala Total \\
\hline Desplazamientos largos & $\begin{array}{l}13,99 \pm 4,58 \\
14,20 \pm 4,56 \\
\text { t:- } 0,616 \\
\text { p: } 0,538\end{array}$ & $\begin{array}{l}9,75 \pm 3,42 \\
10,26 \pm 3,73 \\
\text { t: }-1,870 \\
\text { p: } 0,062\end{array}$ & $\begin{array}{l}23,74 \pm 6,93 \\
24,46 \pm 7,09 \\
\text { t: }-1,356 \\
\text { p: } 0,175\end{array}$ \\
\hline Compaginar estudios y trabajo & $\begin{array}{l}14,33 \pm 4,60 \\
13,48 \pm 4,43 \\
\text { t: } 2,239 \\
\text { p: } 0,025\end{array}$ & $\begin{array}{l}10,10 \pm 3,64 \\
9,74 \pm 3,43 \\
\text { t: } 1,208 \\
\text { p: } 0,228\end{array}$ & $\begin{array}{l}24,42 \pm 7,13 \\
23,22 \pm 6,63 \\
\text { t: } 2,076 \\
\text { p: } 0,038\end{array}$ \\
\hline Otras obligaciones (cuidar hijos, padres,...) & $\begin{array}{l}14,00 \pm 4,60 \\
14,74 \pm 4,27 \\
\text { t: }-1,434 \\
\text { p: } 0,152\end{array}$ & $\begin{array}{l}9,84 \pm 3,50 \\
11,09 \pm 3,80 \\
\text { t:- } 3,107 \\
\text { p: } 0,002\end{array}$ & $\begin{array}{l}23,85 \pm 7,02 \\
25,83 \pm 6,65 \\
\text { t: }-2,523 \\
\text { p: } 0,012\end{array}$ \\
\hline Asignaturas pendientes cursos anteriores & $\begin{array}{l}13,49 \pm 4,42 \\
15,35 \pm 4,61 \\
\text { t:-5,165 } \\
\mathrm{p}<0,001\end{array}$ & $\begin{array}{l}9,84 \pm 3,45 \\
10 ., 13 \pm 3,50 \\
\text { t:- } 1,750 \\
\text { p: } 0,081\end{array}$ & $\begin{array}{l}23,32 \pm 6,80 \\
25,69 \pm 7,14 \\
\text { t: }-4,254 \\
p<0,001\end{array}$ \\
\hline Reside con la familia durante el curso & $\begin{array}{l}14,00 \pm 4,43 \\
14,24 \pm 4,77 \\
\text { t:- } 0,672 \\
\text { p: } 0,502\end{array}$ & $\begin{array}{l}9,89 \pm 3,63 \\
10,13 \pm 3,92 \\
\text { t:- }-0,871 \\
\text { p: } 0,384\end{array}$ & $\begin{array}{l}23,89 \pm 6,94 \\
24,37 \pm 7,12 \\
\text { t: }-0,883 \\
\text { p: } 0,378\end{array}$ \\
\hline $\begin{array}{r}\text { Hombre } \\
\text { Mujer }\end{array}$ & $\begin{array}{l}13,86 \pm 4,56 \\
14,31 \pm 4,57 \\
\text { t:- } 1,296 \\
\text { p: } 0,195\end{array}$ & $\begin{array}{l}10,04 \pm 3,61 \\
9,95 \pm 3,55 \\
\text { t: } 0,325 \\
\text { p: } 0,745\end{array}$ & $\begin{array}{l}23,89 \pm 7,12 \\
24,25 \pm 6,90 \\
\text { t: }-0,678 \\
\text { p: } 0,498\end{array}$ \\
\hline $\begin{array}{r}\text { C. Sociales y Comunicación } \\
\text { Ciencias Jurídicas y Empresa } \\
\text { Escuela Politécnica } \\
\text { Ciencias Salud y del Deporte }\end{array}$ & $\begin{array}{l}14,26 \pm 4,46 \\
13,75 \pm 4,47 \\
15,05 \pm 4,45 \\
13,31 \pm 4,66 \\
\text { F: } 5,239 \\
\text { p:0,001 }\end{array}$ & $\begin{array}{l}10,09 \pm 3,41 \\
9,70 \pm 3,28 \\
10,58 \pm 3,79 \\
9,56 \pm 3,61 \\
\text { F: } 3,161 \\
\text { p:0,024 }\end{array}$ & $\begin{array}{l}24,34 \pm 6,90 \\
23,45 \pm 6,50 \\
25,63 \pm 7,11 \\
22,87 \pm 7,01 \\
F: 5,845 \\
\text { p:0,001 }\end{array}$ \\
\hline
\end{tabular}

t: Estadístico T de Student $\quad F$ : Estadístico análisis de la varianza $\quad$ p: significación estadística.

que tienen asignaturas pendientes de cursos anteriores, obtienen puntuaciones mayores en la escala total y las dos subescalas, resultando estas diferencias estadísticamente significativas.

Validez de Criterio: En la tabla 5 se puede observar que tanto los valores de las dos subescalas como la escala total presentan una correlación positiva con los cuestionarios de ansiedad y depresión de Goldberg. La correlación más elevada corresponde a la variable "Total escala" con la escala de depresión de Goldberg, R: 0,464 ( $\mathrm{p}<0,001)$, muy parecida a la que ofrece con la escala de ansiedad, R: $0,419(\mathrm{p}<0,001)$. Los trastornos depresi-
Tabla 5

Análisis de Correlación entre las escalas del cuestionario y las puntuaciones del cuestionario de Goldberg

\begin{tabular}{|l|c|c|c|c|c|}
\hline & Ansiedad & Depresión & $\begin{array}{c}\text { Aspectos } \\
\text { académicos }\end{array}$ & $\begin{array}{c}\text { Aspectos } \\
\text { socio } \\
\text { familiares }\end{array}$ & $\begin{array}{c}\text { Total } \\
\text { escala }\end{array}$ \\
\hline Ansiedad & 1 & $\begin{array}{c}0,602 \\
\mathrm{p}<0,001 \\
\mathrm{~N}: 674\end{array}$ & $\begin{array}{c}0,339 \\
\mathrm{p}<0,001 \\
\mathrm{~N}: 700\end{array}$ & $\begin{array}{c}0,387 \\
\mathrm{p}<0,001 \\
\mathrm{~N}: 700\end{array}$ & $\begin{array}{c}0,419 \\
\mathrm{p}<0,001 \\
\mathrm{~N}: 700\end{array}$ \\
\hline Depresión & & 1 & $\begin{array}{c}0,392 \\
\mathrm{p}<0,001 \\
\mathrm{~N}: 674\end{array}$ & $\begin{array}{c}0,406 \\
\mathrm{p}<0,001 \\
\mathrm{~N}: 674\end{array}$ & $\begin{array}{c}0,464 \\
\mathrm{p}<0,001 \\
\mathrm{~N}: 674\end{array}$ \\
\hline $\begin{array}{l}\text { Aspectos } \\
\text { académicos }\end{array}$ & & $\mathrm{N}: 674$ & 1 & $\begin{array}{c}0,471 \\
\mathrm{p}<0,001 \\
\mathrm{~N}: 700\end{array}$ & $\begin{array}{c}0,893 \\
\mathrm{p}<0,001 \\
\mathrm{~N}: 700\end{array}$ \\
\hline $\begin{array}{l}\text { Aspectos } \\
\text { sociofamiliar }\end{array}$ & & & $\mathrm{N}: 700$ & 1 & $\begin{array}{c}0,818 \\
\mathrm{p}<0,001 \\
\mathrm{~N}: 700\end{array}$ \\
\hline Total escala & & & & $\mathrm{N}: 700$ & $\begin{array}{c}1 \\
\mathrm{~N}: 700\end{array}$ \\
\hline
\end{tabular}

p: Sinificación estadística. 
Tabla 6

Valores medios de la escala y subescala en función de la existencia de posibles trastornos de ansiedad o depresión

\begin{tabular}{|c|c|c|c|c|}
\hline & & Aspectos académicos & $\begin{array}{c}\text { Aspectos socio } \\
\text { familiares }\end{array}$ & Escala Total \\
\hline Ansiedad Goldberg & $\begin{array}{l}\text { No } \\
\text { Sí }\end{array}$ & $\begin{array}{l}12,86 \pm 4,0 \\
15,47 \pm 4,69 \\
\text { t: }-7,792 \\
\mathrm{p}<0,001\end{array}$ & $\begin{array}{l}8,89 \pm 3,01 \\
11,23 \pm 3,76 \\
\text { t: }-9,012 \\
p<0,001\end{array}$ & $\begin{array}{l}21,75 \pm 6,12 \\
26,69 \pm 7,02 \\
\text { t: }-9,078 \\
p<0,001\end{array}$ \\
\hline Depresión Goldberg & $\begin{array}{l}\text { No } \\
\text { Sí }\end{array}$ & $\begin{array}{l}12,30 \pm 3,99 \\
15,22 \pm 4,53 \\
t:-8,0672 \\
p<0,001\end{array}$ & $\begin{array}{l}8,75 \pm 2,85 \\
10,83 \pm 3,73 \\
\mathrm{t}:-8,068 \\
\mathrm{p}<0,001\end{array}$ & $\begin{array}{l}21,05 \pm 5,74 \\
26,04 \pm 6,99 \\
t:-9,999 \\
p<0,001\end{array}$ \\
\hline
\end{tabular}

t: Estadístico T de Student $\quad$ F: Estadístico análisis de la varianza $\quad$ p: significación estadística.

vos, en todos los casos, están más fuertemente correlacionados con la escala y subescalas del estudio que los trastornos de ansiedad, siendo siempre estas correlaciones estadísticamente muy significativas, $\mathrm{p}<0,001$.

En la tabla 6 aparecen los resultados de los valores medios de la escala total y de las dos subescalas, en función de la existencia de ansiedad o depresión, resultando en todas ellas una puntuación siempre superior en los alumnos que presentaban afirmativos el cuestionario de depresión y de ansiedad de Goldberg, siendo las diferencias encontradas estadísticamente muy significativas, $\mathrm{p}<0,001$.

\section{DISCUSIÓN}

El desarrollo de herramientas que permitan conocer otros aspectos asociados a los trastornos de ansiedad y depresión como posibles factores predictores de estos procesos, cobra gran interés en la medida en que resulta posible la detección precoz según otras perspectivas.

En la mayoría de ocasiones, esta información llega al profesorado debido a la relación que se establece con el alumnado. Este aspecto brinda al profesor y/o tutor una posición privilegiada para poder enfocar esa información sobre circunstancias de la vida del estudiante con fines preventivos.
En este sentido, señalamos la importancia que debe darse a la identificación y concomitancia de los aspectos sociofamiliares y académicos señalados en nuestro estudio para poder intuir situaciones de riesgo a tiempo.

La fiabilidad del cuestionario medida con el test-retest obtuvo un coeficiente de correlación bastante elevado, 0,819, valor superior al recomendado por otros autores, lo que nos indica que el cuestionario presenta una buena reproducibilidad y estabilidad en el tiempo ${ }^{32}$.

La consistencia interna del cuestionario global y la subescala resultante del análisis factorial, "aspectos académicos" es bastante aceptable, $\alpha: 0,762$ y $\alpha: 0,711$, supera sensiblemente el valor recomendado de ser siempre $>0,700$, no ocurriendo así con la subescala "aspectos socio familiares", $\alpha: 0,608$, pero entra como valores aceptables en este tipo de escalas valores mas bajos, entre 0,50 y 0,60 , cuando uno de sus objetivos es establecer comparaciones entre grupos poblacionales y en procesos iniciales de investigación sobre la eficacia del instrumento a valorar ${ }^{35,36}$.

El estudio realizado sobre la validez predictiva del cuestionario indica una relación significativa con las puntuaciones de la escala Goldberg, pudiendo así constituir un buen indicador de validez predictiva del cuestionario y contribuyendo a confirmar la 
validez global del instrumento. En relación a los instrumentos existentes, existen diversas herramientas que valoran tanto fuentes de ansiedad estrictamente del ámbito académico ${ }^{39}$, como aspectos totalmente independientes con acción indirecta sobre el nivel de estrés. La característica que presenta nuestro instrumento validado es que combina diversos aspectos relacionados con la ansiedad y depresión, tanto académicos como otros aspectos personales y sociofamiliares, de manera que podría realizarse en fases posteriores de la investigación, un análisis comparativo con los resultados de otras escalas con fines parecidos para determinar posibles mejoras.

Nuestro estudio cuenta con una muestra representativa y los hallazgos estadísticos están respaldados por elevado nivel de significación, lo que permite poder extrapolar los resultados a la población de estudio, y por tanto, confirmar la validación del instrumento, no siendo en nuestro caso el aspecto de la muestra un aspecto limitante. Por otro lado, cabe tener en cuenta que la escala Goldberg es una herramienta utilizada como técnica de screening, principalmente en el entorno sanitario, no siendo en sí misma un instrumento diagnóstico sino de detección precoz de posibles casos.

Con este estudio, se consigue demostrar la elevada consistencia interna y la validez del cuestionario de creación propia sobre situaciones sociofamiliares y académicas que pueden estar relacionadas con alteraciones ansioso/depresivas, utilizando como referencia el cuestionario de Goldberg para la validez de criterio, siendo una herramienta válida, fiable y que proporciona una medida útil para la detección precoz de ansiedad y depresión.

La principal aportación que realizamos con este instrumento es señalar algunas preguntas, para la posible identificación de estudiantes con situación de riesgo para estos trastornos a raíz del conocimiento por parte de los profesores y/o tutores del ámbito académico, de una serie de aspectos de su vida, relativos a actividades laborales, cargas familiares, situación académica, entorno familiar, etc. sobre todo teniendo en cuenta que en la actualidad no se ha establecido en la mayoría de Universidades una sistemática de aplicación de cribajes al uso para tales fines.

Esta herramienta de valoración, de fácil uso y ágil aplicación sobre situaciones sociofamiliares y académicas, plantea posibles beneficios en cuanto a la detección precoz y la prevención de psicopatologías y otros trastornos. El elevado nivel de riesgo de sufrir ansiedad y depresión en el entorno universitario se demuestra actualmente por las cifras de estudiantes que se ven afectados por estos trastornos (constatados normalmente a posteriori). Son por tanto las actividades de carácter eminentemente preventivo encaminadas a la disminución de la incidencia de estos trastornos en dicha población las que deben potenciarse y mejorarse, siendo el entorno universitario el que presenta mayor accesibilidad a las poblaciones de estudiantes.

\section{BIBLIOGRAFÍA}

1. Fisher S, y Hood R. The stress of the transition to university: a longitudinal study of vulnerability to psychological disturbance and home-sickness. $\mathrm{Br}$ J Psychol. 1986; 79: 1-13.

2. Polo A, Hernández JM y Pozo C. Evaluación del estrés académico en estudiantes universitarios. Ansiedad estrés. 1996; 2 (2/3): 159-172.

3. Pellicer O, Salvador A, y Benet I. A., Efectos de un estresor académico sobre las respuestas psicológica e inmune en jóvenes. Psicothema. 2002; 14 (2): 317-322.

4. Martín IM. Estrés académico en estudiantes universitarios. Apuntes Psicol 2007; 25 (1): 87-99.

5. Riveros M, Hernández H. y Rivera J. Niveles de depresión y ansiedad en estudiantes universitarios de Lima Metropolitana. Rev Inv Psicol. 2007; 10 (1): 92-102. 
6. Perales A., Sogi C. y Morales R., Estudio comparativo de salud mental en estudiantes de medicina de dos universidades estatales peruanas. An Fac med Univ nac mayor San Marcos. 2003; 64 (3): 239-246.

7. Tavecchio LWC, Thomer MAE y Meeus W, Attachment, social network ad homelessness in young people. Soc Behav Pers. 1999, 27 (3): 247263.

8. Seibel FL y Brad JW, Parental control, trait anxiety and satisfaction with life in college students. Psychol Rep. 2001, 88 (2): 473-481.

9. Chase-Lansdale PL y Cherlin, A J, The long-term effects of parental divorce on the mental health of young adults: a developmental perspectiva, Child Dev. 1995; 66 (6): 1614-1634.

10. Hall LA, Parental bonding: a key factor for mental health of college women, Issues Ment Health Nurs. 2004; 25 (3): 277-291.

11. Johnson HD, LaVoie JC y Mahoney M, Interparental conflict and family cohesion: predictors of loneliness, social anxiety and social avoidance in late adolescence. J Adolescent Res. 2001; 16 (3): 304-319.

12. Lee RM, Su J, y Yoshida E, Coping with intergeneracional family conflict among asian american collage students. J Cou Psychol. 25 (3): 389-399.

13. Tan S, y Rey J. Depression in the young, parental depression and parenting stress, Aust N Z J Psychiatr. 2005;13 (1): 76-79.

14. Lizardi H, Psychopathology in the adolescent and young adult offspring of parents with dysthymic disorder and major depressive disorder, J Nerv Ment Dis. 2004; 192 (3): 193-199.

15. Klein D, et al, Psychopathology in the adolescent and young adult offspring of a community simple of mothers and fathers with major depression, Psychol Med. 2005; 35 (3): 353-365.

16. Lewinsohn PM, Olino, TM. y Klein D, Psychosocial impairment in offspring of depressed parents, Psychol Med. 2005; 35 (10): 1493-1503.

17. D'Zurilla TJ, Sheedy C F, Relation between social problem-solving ability and subsequent level of psychological stress in college students, J Pers Soc Psychol. 1991; 61 (5): 841-846.

18. Rubio ChT, y Lubin B, Collage student mental health: a person-environment interactional analysis, J Clin Psychol. 1986; 42 (1): 205-212.
19. Overbeek G, et al, Young adults' relationship transition and the incidence of mental disorders: a three-wave longitudinal study, Soc Psychiatry Epidemiol. 2003; 38 (12): 669-676.

20. Friis RH, Wittchen HU, Pfister H, Lieb R. Life events and changes in the course of depression in young adults. Eur Psychiatry. 2002; 17 (5): 241253.

21. Edwards KJ. Stress, negative social exchange and health symptoms in university students. J Am Coll Health. 2001;50 (2): 75-80.

22. Carney C, McNeish S, y McColl J. The impact of part time employment on students' health and academic performance: a Scottish perspective, J F High Ed. 2005;29 (4): 307-319.

23. Roberts R, y Goldgin J. The effects of economic circumstances on British Student: Mental and physical health, J Am Coll Health. 1999; 48 (3): 103-110.

24. Lawyer SR, Kart RS, Murphy JG, McGlynn F. Heavy drinking among college students is influende by anxiety sensitivity, gender, and contexts for alcohol use, J Anxiety Disorders 2002;16 (2): 165-169.

25. Caldwell TM, Rodgers B, Jorm AF, Christensen H, Jacomb PA, Korten AE, Lynskey MT. Patterns of association between alcohol consumption and symptons of depression and anxiety in young adults, Addiction. 2002; 97 (5): 583-595.

26. Valentier D, Mounts NS y Deacon BJ. Panic attacks, depresión and anxiety symtoms, and substance use behaviors during late adolescente. J Anxiety Disorders. 2004; 18 (5): 573-585.

27. Weitzman ER, Poor mental, depression and associations with alcohol consumption, harm, and abuse in a national sample of young adults in college. J Nerv Ment Dis 2004;192 (4): 269-277.

28. Zimmermann P, Wittchen HU, Höfler M, Pfister H, Kessler RC, Lieb R. Primary anxiety disorders and development of subsequent acohol use disordes: a 4-year community study of adolescents and young adults, Psychol Med 2003; 33 (7): 1211-22.

29. Miller P, Surtees PG. Psychological symptoms and their course in first-year medical students as assessed by interval general heath questionnaire (I-GHQ). Br J Psychiatry. 1991; 159: 199-207.

30. Guthrie EA, Black D, Shaw CM, Hamilton F, Creed FH, Tomerson B. Embarking upon a medical career: psychological morbility in first year medical students. Med Educ. 1995; 29:337-341. 
31. Argimón Pallás JM, Jiménez Villa J. Métodos de investigación en clínica y epidemiología. $3^{\mathrm{a}}$ ed. Madrid: Elsevier; 2004, pp.196-207.

32. Streiner DL, Norman GR. Health measurement scales. A practical guide to their development and use. Mew York: Oxford University Press; 1989.

33. Cronbach LJ. Coefficient alpha and internal structure of test. Psychometrical. 1951; 16:297-334.

34. Chinn S, Burney P. On measuring repeatability of data from self-administered questionnaires. Int $\mathrm{J}$ Epidemio. 1987; 16:121-7.

35. Stewart AL, Ware JF. Measuring functioning and well-being. The medical outcome study approach. Durham: Duke University Press; 1992, pp. 76-81.
36. Nunally J. Psychometric theory. $2^{\text {nd }}$ ed New York: McGraw-Hill; 1978.

37. Goldberg D, Bridges K, Duncan-Jones P, et al. Detecting anxiety and depression in general medical settings. Br Med J. 1988; 97: 897-899.

38. Related Articles, LinksGoldberg D, Bridges K, Duncan-Jones P, Grayson D. Detección de la ansiedad y la depresión en el marco de la medicina general. Br Med J. (ed. esp.) 1989; 4(2): 49-53.

39. De Pablo J, Baillés E, Pérez J y Valdés M. Construcción de una escala de estrés académico para estudiantes universitarios. Educ Med. 2002; 5 (1): 46-52. 